\title{
Effects of lavender oil inhalatic
on sternotomy related pain intensity in open heart surgery patients in Egypt
}

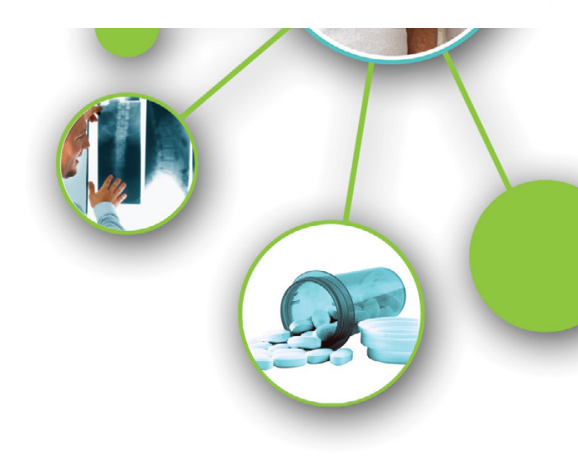

\begin{abstract}
Background: Sternotomy related pain is the main patients' frequent complaint after open heart surgery. Because of the deleterious effects of opioids, it is important to use an alternative way to effectively reduce pain including aromatherapy with analgesics.
\end{abstract}

Aim of the study: This study aimed to evaluate the effectiveness of inhaled lavender oil on an intensity of sternotomy related pain and vital signs in patients who underwent open heart surgery.

Subjects and Methods: Quasi-experimental research design was conducted on a convenient sample of 90 patients recruited from 9-bed open heart surgery intensive care unit affiliated to Al Manial university hospital and then assigned randomly to study and groups. The study group was exposed to lavender inhalation with morphine. While the control group of patients exposed to morphine only. Two questionnaires were utilized in data collection. The first one included baseline characteristics including demographic and medical data and the second one were as a visual analog scale to determine the pain intensity.

Results: Significant statistical difference was found between the study and control group as the study group demonstrated lower pain intensity scores than the control group (at 5 minutes and, half an hour after).

Recommendations: Utilize lavender oil inhalation in the early days of open heart surgery to decrease the consumption of opoids and analgesics.

Keywords: Inhalation, lavender oil, sternotomy, related pain, open heart surgery

\section{Introduction}

Inadequate management of pain after surgeries continues to be a major drawback and leads to adverse effects. Proper and adequate management of postoperative pain requires indepth knowledge of the pathological alterations that happens throughout pain, appropriate pain management methods to alleviate pain, exacerbating factors such as anxiety and fears utilizing a variety of pharmacological and nonpharmacological methods after surgery improve postoperative pain management 1 , provide adequate pain relief, decrease the hazards, and enhance patient comfort [1].

Despite the technological advances in open heart surgery, patients who undergo these surgeries usually complain of major complications after surgery including, dysrhythmia, cardiologic shock, pain, delirium, and vital sign compromise. Many factors may might worsen mental stress in patients admitted to intensive care units including pain, insomnia, and anxiety due to being far away from home and work [2,3]. Preoccupying with surgery may result in overstimulation of the sympathetic response such as rapid respiration, rapid pulse, decreased body temperature, elevated blood pressure and decreased tissue perfusion [4] Mental stress and vital signs alterations may potentiate postoperative pain and increased consumption of analgesics indirectly, as well as decreasing resistance to infection, delaying wound healing [5] and increasing the duration of hospitalization [6].

Lack of pain management in acute postoperative patients is associated with complications such as exaggerated morbidity,
Nahla Shaaban Khalil ${ }^{1 *}$, Manal Sayed Ismaeel ${ }^{1,2}$, Sherine Ali Hassan ${ }^{1,3}$, Hesham shawky ${ }^{4}$

${ }^{1,2}$ Critical care and Emergency Nursing, faculty of Nursing, Cairo University, Egypt

${ }^{3}$ Registered nurse, Tanta University Hospital, Egypt

${ }^{4}$ Cardiothoraci Surgery

Department, Cairo University, Egypt

*Author for correspondence:

nahlakhalil28@yahoo.com 
restricted physical activity, delayed recovery, opioid dependence during hospitalization, and increased medical costs. Opioids administration are still the traditional and preferred management of acute postoperative pain, However, the side effects made by opioid- can restrict adequate dosing for relieving pain leading to more worse pain after surgery [7].

Utilizing non-pharmacological pain management methods beside analgesics may alleviate the pain, embarrassment, and harmful side-effects. Moreover, they are considered as safe, being low cost and easy to use. Furthermore, they are reported to be acceptable and bearable by patients. One of the complementary pain relief therapeutic measures is known as aromatherapy. Using this therapy is easy and economical when compared to other nonpharmacological techniques such as reflexology, and transdermal electrical stimulation, acupuncture. Also, it does not need any medical consultant $[8,9]$. Aromatherapy is a technique where essential oils are utilized for inhalation [10] which can relieve pain, and stabilize the hemodynamics [11,12]. One of the examples of aromatherapy is Lavender, it an essential oil that is used in a broad way in various fields, the medical properties of Lavender include its action as an analgesic, as a narcotic, sedative, anti-inflammatory, and anti-depressive agent [13]. Lavender improves the heart function and promoting blood supply with amenable effects on coronary circulation [14]. Cardiac surgery is usually associated with pain, emotional upset and unstable hemodymamics. Therefore critical care nurses have significant roles in assessing and managing pain and vital signs alterations as well as implementing appropriate nursing interventions and evaluating the beneficial effects. Thus, this study was conducted to evaluate the effects of lavender inhalation oil in reducing sternotomy pain after open heart surgery.

\section{- The aim of the study}

The study aimed to evaluate the effect lavender oil inhalation on the severity of sternotomy related pain in open-heart surgery patients

\section{Subjects and Methods}

\section{- Research design}

The quasi-experimental research design was applied in the present study because it included a broad range of non-randomized intervention studies. These designs are frequently used when it is not feasible to use a randomized, controlled trial [15].

\section{- Setting}

This study was conducted in the intensive care unit of the Cardiothoracic Surgery Department affiliated to Cairo University Hospitals (AL Manial University Hospital).

\section{- Participants}

Random assignments of ninety patients who had open heart surgery at Cairo university hospitals and consented to participate in the study were recruited. Then, these patients were distributed equally and randomly according to surgery schedule to the study and control groups. The inclusion criteria included patients who were more than 18 years, extubated and fully conscious for 24 hours after surgery, oriented to time and place, and had voluntary breathing. On the other hand, exclusion criteria included patients who had allergies or required medical intervention during the inhalation session, psychological disorders and respiratory problems, history of head injury or convulsion, disturbed smelling function, using anxiolytic drugs, and intubation for more than 24 hours.

\section{- Data collection tools}

The data collection instruments included patients' baseline characteristics, medical related data and Visual Analog Scale (VAS) for evaluating the pain intensity. A visual analog scale is a common standardized tool [16] like a $10 \mathrm{~cm}$ ruler including 10 numbers beginning from 0 (no pain) and ending at 10 (most severe pain). Various cases of a human face in response to pain severity have been outlined on the opposite side of the ruler. The patients were requested to choose one of faces according to their pain severity. The number illustrated on the back of the ruler was estimated.

\section{- The procedure of data collection}

The overall study was approved by the Research Ethics Committee of the faculty of nursing at Cairo University and the protocol was registered by ethical Clinical Trials (No. FWA 00019803). Later, official permission was obtained from the hospital authorities at 
El Manial University Hospital. Later, two days before surgery, all the waiting list of patients who met the inclusion criteria were divided randomly into study and control groups. The study group was given odd number during random selection in the checklist and the control group was given even number. Later, the researchers collected and documented the demographic and medical data. During the phase of data collection, the researchers explained the nature of the study to their patients individually and emphasized that participating in that study was absolutely optional and they had the right to quit from the study at any time without rationale.

Next, before initiating the interventions in both groups, the patients were contacted for clarifying the manner of the use of VAS to estimate the score of pain severity accurately among patients. In the afternoon shift of the first and second days after surgery, the control group of patients was contacted by the researchers to evaluate the severity of pain making use of VAS before opioid administration. Later, the control group was received the prescribed opioid drugs (morphia sulfate). As well, the study group of patients was approached by the researchers to evaluate the severity of pain utilizing VAS before opioid administration. Later, the study group received opioid (morphia sulfate) followed immediately by patients' inhalation of a piece of cotton saturated with 2 drops of lavender oil that was put in their oxygen face mask for ten minutes. Evaluation of pain intensity was evaluated at four-time frames before, five minutes, 30 minutes, and 2 hours after interventions for both groups.

\section{Data analysis}

Statistical Package of Social Sciences (SPSS) program version 20 was utilized. Descriptive statistics were used like frequencies and percentage distribution to illustrate the demographic and medical data. Moreover, inferential statistics were utilized such as t-test and ANOVA for the comparison of the pain scores among the study and control group.

\section{Results}

Two-thirds of patients were males in both the study and control group with the mean age of 48 years. Both groups had underlying diseases such as diabetes and hypertension. Moreover, no significant difference were found concerning age $(\mathrm{p}=0.2)$, gender $(\mathrm{p}=1.27)$, smoking $(\mathrm{p}=1.6)$, and underlying diseases such as diabetes $(\mathrm{p}=0.52)$, and hypertension $(\mathrm{p}=0.52)$ between two groups (TABLE 1).

Comparison of mean pain scores between the study and control groups revealed significant differences before, 5 minutes after, and 30 minutes after $(p<0.001)$ while no differences were found between them at 2 hours after $(\mathrm{p}=0.22)$. In addition, one way ANOVA analysis for the comparison of means of pain showed

\begin{tabular}{|c|c|c|c|c|c|c|c|c|}
\hline & \multicolumn{2}{|c|}{ Study G } & \multicolumn{2}{|c|}{ Control G } & \multicolumn{2}{|c|}{ Total } & \multicolumn{2}{|c|}{ Chi-square } \\
\hline & $\mathbf{N}$ & $\%$ & $\mathbf{N}$ & $\%$ & $\mathbf{N}$ & $\%$ & $\mathbf{X 2}$ & P-value \\
\hline \multicolumn{9}{|l|}{ Age } \\
\hline 20-29 & 3 & 6.7 & 1 & 2.2 & 4 & 4.4 & \multirow{3}{*}{4.286} & \multirow{3}{*}{0.232} \\
\hline 30-39 & 6 & 13.3 & 2 & 4.4 & 8 & 8.9 & & \\
\hline $40-49$ & 11 & 24.4 & 17 & 37.8 & 28 & 31.1 & & \\
\hline$>49$ & 25 & 55.6 & 25 & 55.6 & 50 & 55.6 & & \\
\hline Mean \pm SD & \multicolumn{2}{|c|}{$47.86 \pm 10.1$} & \multicolumn{2}{|c|}{$50.04 \pm 8.02$} & \multicolumn{2}{|c|}{$48.95 \pm 9.14$} & & \\
\hline \multicolumn{9}{|l|}{ Gender } \\
\hline female & 17 & 37.8 & 12 & 26.7 & 29 & 32.2 & \multirow{2}{*}{1.272} & \multirow{2}{*}{0.259} \\
\hline Male & 28 & 62.2 & 33 & 73.3 & 61 & 67.8 & & \\
\hline \multicolumn{9}{|l|}{ Smoking } \\
\hline No & 24 & 53.3 & 18 & 40 & 42 & 46.7 & \multirow{2}{*}{1.607} & \multirow{2}{*}{0.205} \\
\hline Yes & 21 & 46.7 & 27 & 60 & 48 & 53.3 & & \\
\hline \multicolumn{9}{|c|}{$\begin{array}{l}\text { Underlying diseases } \\
\text { DM }\end{array}$} \\
\hline No & 25 & 55.6 & 22 & 48.9 & 47 & 52.2 & \multirow{2}{*}{0.401} & \multirow{2}{*}{0.527} \\
\hline Yes & 20 & 44.4 & 23 & 51.1 & 43 & 47.8 & & \\
\hline \multicolumn{9}{|l|}{ HTN } \\
\hline No & 20 & 44.4 & 17 & 37.8 & 37 & 41.1 & \multirow{2}{*}{0.413} & \multirow{2}{*}{0.52} \\
\hline Yes & 25 & 55.6 & 28 & 62.2 & 53 & 58.9 & & \\
\hline
\end{tabular}




\begin{tabular}{|c|c|c|c|c|}
\hline pain scale & Study group $(n=45)$ & Control group $(n=45)$ & & \\
\hline Time & Mean \pm SD & Mean \pm SD & $\mathbf{t}$ & P-value \\
\hline Pre & $6.22 \pm 1$ & $7.76 \pm 0.88$ & 7.721 & $<0.001^{* *}$ \\
\hline 5 minutes after & $4.58 \pm 1.23$ & $6.84 \pm 1$ & 9.578 & $<0.001^{* *}$ \\
\hline 30 min after & $4.58 \pm 1.23$ & $6.84 \pm 1$ & 9.578 & $<0.001^{* *}$ \\
\hline \multirow[t]{3}{*}{$2 \mathrm{hs}$ after } & $5.04 \pm 1.28$ & $5.33 \pm 0.95$ & 1.215 & 0.228 \\
\hline & $\mathrm{F}=19.05$ & $F=49.59$ & & \\
\hline & $p=0.0000$ & $\mathrm{P}=0.000$ & & \\
\hline
\end{tabular}

significant differences in the four times of measurement among the study $(\mathrm{P}=0.000)$ and control groups $(\mathrm{P}=0.000)$ when compared before, after 5 minutes, after 30 minutes, and after 2 hours. The highest differences were obvious between before and both 5 minutes, 30 minutes, and 2 hours after in the study and control groups (TABLE 2).

\section{Discussion}

In the present study, the researchers detected that inhalation of lavender oil had significant effects on severity of sternotomy related pain in patients who had undergone open-heart surgery. Truly, the mean pain scores of the patients in study group groups decreased when compared to the control group notably after 5 and 30 minutes of intervention. On the contrary, the control group had higher mean pain scores at the same time after 5 and 30 minutes. Moreover, no significant variations were found between both groups after two hours. This finding may have relevance to the enhancing effect of lavender oil inhalation when administered directly after opioid medication. Supporting to our findings, another similar study done by Sasannejad, Saeedi, and Shoeibi [17] stated that the inhalation of Lavender oil is who proposed to be effective and safe treatment in acute management of headache induced by migraine and mentioned significant reduction of pain severity and associated symptoms after 15 minutes inhalation of lavender oil through rubbing of two to three drops of the lavender essential oil on patients' upper lips in the early onsets of pain.

Likewise, the pain-reducing effects of lavender oil have been extensively Studied by previous studies done [18-20]. It has been illustrated that lavender oil can decrease the pain associated with different circumstances like labor, needle insertion, dialysis and headache [21,22]. From a physiological view that was explained by Re, Barocci, Sonnino, Mencarelli, and Vivani
[23] that an element of Linalool in lavender could inhibit the release of acetylcholine, which changes the functions related to the channels of ions on the neuromuscular synapses. Moreover, Linalyl acetate component has a narcotic effect and linalool works as a sedative agent. Accordingly, these functions rationalize the pain-reducing effect of lavender.

In agreement with our findings, [24] who studied the effectiveness of lavender essence on sternotomy related pain intensity after coronary artery bypass surgery revealed that pain decreased following the inhalation of lavender when compared to control group who did not take lavender. Similarly, our finding is agreed partially with another study done by [25] who suggested that Lavender decreases significantly the pain during the early times of deliveries in women. As well, the current finding is supported by another study done by [26,27] who mentioned that administration of inhaled Lavender through an oxygen face mask can be utilized to decrease the need for analgesics and opioids in immediate postoperative period of breast biopsy and in patients undergoing laparoscopic surgeries.

On the contrary, our study findings contradicted with a previous study conducted by Kim [26] who detected the pain-relieving effects of Lavender in patients who inhaled Lavender $2 \%$ mixed with oxygen via oxygen mask after operating breast biopsy had more satisfaction in relation to perceived relief of pain, but no changes were seen in perceived pain in the control group. Moreover, Shahnaz and colleagues [28] revealed that inhaling lavender oil had no effect on pain severity and only reduced the anxiety of study group.

There is a lack of research concerning the effect of Lavender oil inhalation on the pain severity following sternotomy and on the nursing roles in the administration of this therapy. This type of therapy is considered the 
art of nursing, which along with science, could provide better and more efficient nursing care. Therefore, the researchers aimed to implement that intervention to evaluate the effects of Lavender oil inhalation on the severity of sternotomy related pain after open heart surgery to attain the patient's satisfaction and improve the patients' outcomes and consequently decrease the medical expenses [29].

\section{Conclusion}

Inhalation of lavender oil can be used in combination with analgesics /opioids to relieve pain in patients after open heart surgery, in order to reduce the consumption of narcotics and nonnarcotic drugs and their deleterious effects.

\section{Recommendation}

Based on the findings of the present study, the following recommendations are suggested -Utilize lavender oil inhalation in the early days of open-heart surgery to decrease the consumption of opioids and analgesics.

\section{Acknowledgments}

This article was a part of the of Master's thesis in critical care Nursing, and Emergency Department registered at Faculty of Nursing, Cairo University It was approved by the (No. No. FWA 00019803) at Ethics Committee of the faculty of nursing Cairo university. Our sincere appreciation offered to all open-heart surgery patients at cardiothoracic surgery department at al Manual university Hospital who participating in this study. The authors wish to express their deepest gratitude to all people who shared or contributed in this valuable work.

\section{References}

Lovich Sapola J, Smith CE, Brandt CP. Postoperative pain control. Surg Clin North Am. Apr. 95 (2), 301-318 (2015).

Gallagher R, Mckinley S.H. Stressors and anxiety in patients undergoing coronary artery bypass surgery. Am J Crit Care. 16(3), 248-577 (2007).

Bauer BA, Cutshall SM, Wentworth $L J$, et al. Effect of massage therapy on pain, anxiety, and tension after cardiac surgery: a randomized study. Complement Ther Clin Pract. 16(2), 53-70 (2010).

Barker R, Kober A, Hoerauf $\mathrm{K}$, et al. Out-of-hospital auricular acupressure in elder patients with hip fracture: a randomized double-blinded trial. Acad Emerg Med. 13(1), 19-238 (2006).

Granot M, Goldstein FS. The roles of pain catastrophizing and anxiety in the prediction of postoperative pain intensity: a prospective study. Clin J Pain. 21(5), 439-459 (2005).

Kalkman CJ, Visser K, Moen J, et al. Preoperative prediction of severe postoperative pain. Pain. 105(3), 4152310 (2003).
Grigoras A, Lee P, Sattar F. Preoperative intravenous lidocaine decreases the incidence of persistent pain after breast surgery. Clin J Pain. 28(7), 567-572 (2012).

Sobhani A, Sharemi H, Rangpur R. Effect of levander on post-cesarean pain. J Guillen Univ Med Sci. 16, 80-86 (2007).

Lehrner J, Marwinski G, Lehr S, et al. Ambient odors of orange and lavender reduce anxiety and improve mood in a dental office. Physiol Behav. 15, 92-95 (2005).

Hur MH, Lee MS, Kim C. Aromatherapy for treatment of hypertension: a systematic review. J Eval Clin Pract. 18(1), 37-41 (2012).

Kim S, Kim HJ, Yeo JS, et al. The effect of lavender oil on stress, bispectral index values, and needle insertion pain in volunteers. J Altern Complement Med. 17(9), 823-615 (2011).

Fayazi S, Babashahi M, Rezaei M. The effect of inhalation aromatherapy on anxiety level of patients in the preoperative period. Iran J Nurs Midwifery Res. 16, 278-8312 (2011).

Chu CJ, Kemper KJ. Lavender The lang wood herbal task force the center for holistic pediatric education and research. Revised July 2, 2001. [Last accessed on Sep 15, 2013]. Available from: http:// www.mcp.edu/herbal/

Shiina Y, Funabashi N, Lee K, et al. Relaxation effects of lavender aromatherapy improve coronary flow velocity reserve in healthy men evaluated by transthoracic Doppler echocardiography. Int J Cardiol. 129(2), 193-197.6 (2008).

Shadish William R, Donald T, Campbell. Experimental and QuasiExperimental Designs for Generalized Causal Inference. Belmont, CA: Wadsworth Cengage Learning (2002).

Warden V, Hurley AC, Volicer L. Development and psychometric evaluation of the Pain Assessment in Advanced Dementia (PAINAD) scale. $J$ Am Med Dir Assoc. 4(1), 9-15 (2003).

Sasannejad P, Saeedi M, Shoeibi A, et al. Lavender essential oil in the treatment of a migraine headache: A placebocontrolled clinical trial. Eur Neurol. 67, 288-291 (2012).

Morris N. The effects of lavender 
(Lavendula Angustifolia) baths on psychological well-being: two exploratory randomized control trials. Complement Ther Med. 10, 223-228 (2002).

Gedney JJ, Glover TL, Fillingim RB. Sensory and affective pain discrimination after inhalation of essential oils. Psychosom Med. 66, 599-606 (2004).

Yip YB, Tse SH. An experimental study on the effectiveness of acupressure with aromatic lavender essential oil for sub-acute, non-specific neck pain in Hong Kong. Complement. Ther Clin Pract. 12, 18-26 (2006).

Olapour A, Behan K, Akhondzadeh $\mathrm{R}$, et al. The effect of inhalation of aromatherapy blends containing lavender essential oil on cesarean postoperative pain. Anesth Pain. 3, 204-207 (2013).

Bagheri-Nesami M, Espahbodi F,
Nikkhah F, et al. The effects of lavender aromatherapy on pain following needle insertion into fistula in the hemodialysis patient. Ther Clin Pract. 20, 1-4 (2014).

Re L, Barocci S, Sonnino, et al. Linalool modifies the nicotinic receptorion channel kinetics at the mouse neuromuscular junction. Pharmacol Res. 42, 177-182 (2000).

Gorji MA, Ashrastaghi OG, Habibi $\mathrm{V}$. The effectiveness of lavender essence on sternotomy related pain intensity after coronary artery bypass grafting. $A d v$ Biomed Res. 4, 127 (2015).

Alavi SM, Farasat Kish R, Sadeghpour Tabaee A., et al. Comparison of the analgesic effect of sufentanil and morphine in patient controlled analgesia method after coronary artery bypass surgery. Iran J Surg. 17, 6-11 (2010).
Kim JT, Wajda M, Cuff G, et al. Evaluation of aromatherapy in treating postoperative pain: Pilot study. Pain Pract. 6, 273-277 (2006).

Kim JT, Ren CJ, Fielding GA, et al. Treatment with lavender aromatherapy in the post-anesthesia care unit reduces opioid requirements of morbidly obese patients undergoing laparoscopic adjustable gastric banding, Obesity Surgery, 17(7), 920-925 (2007).

Shahnazi M, Nikjoo R, Yavarikia P, et al. Inhaled Lavender effect on anxiety and pain caused from intrauterine device insertion. J Caring Sci. 1, 255-261 (2012).

Chaparro LE, Smith SA, Moore RA, et al. Pharmacotherapy for the prevention of chronic pain after surgery in adults. Cochrane Database Syst Rev. (7), 008307 (2013). 